\title{
Surgical Data Science, An Emerging Field of Medicine
}

\author{
Dénes Ákos Nagy*†, Imre J. Rudas* and Tamás Haidegger*† \\ *Antal Bejczy Center for Intelligent Robotics, \\ Óbuda University, Budapest, Hungary \\ ${ }^{\dagger}$ Austrian Center for Medical Innovation and Technology (ACMIT) \\ Wiener Neustadt, Austria \\ Email:\{denes.nagy, imre.rudas, haidegger\}@irob.uni-obuda.hu
}

\begin{abstract}
Computer Assisted Surgery (CAS) significantly changed the course of interventional medicine. The development of medical imaging opened up the possibility for accurate, patient specific planning, and advanced imaging techniques provided the ground for the development of real-time navigation systems. The advancement of minimally invasive surgical techniques and tools required increasing manuality from the surgeon, which facilitated the development of tele-robotic manipulation. These systems provide a vast amount of objective inta-operative data, thus many believe that the next step could be big data analysis for creating and evaluating surgical process models. This emerging field of medicine, called Surgical Data Science, has the potential to improve intervetional medicine with objective statistical analysis, and therefore to provide better patient outcomes and a reduction in healthcare costs.
\end{abstract}

Index Terms-Computer Assisted Surgery; Big Data Analysis; Surgical Process Model; Surgical Data Science

\section{INTRODUCTION}

Traditionally, medicine is considered as a highly hierarchal profession, where decision is mainly based on individual experience, rather than objective measurements. With evidencebased medicine gaining increasing attention in many domains, this approach has already changed several fields of medicine (for example pharmacology and internal medicine) [1].

The same applies to surgery: large scale data from heterogenous sources is becoming available. These include: Electronic Health Record (EHR) systems, digital medical imaging (e.g. Computer Tomography, Ultrasound), computer assisted surgical systems etc. While these data sources are rich in information, they are generally unstructured, therefore they rarely get integrated directly into the surgical workflow. Surgical Data Science (SDS), promises to extract knowledge from these inputs, and provide objective measures, linking treatment decisions to medical evidence. To achieve this goal, SDS aims at creating a framework where data collection, analysis and modelling are linked in a common architecture providing realtime performance feedback to the intervention [2].

Even though many insurance organizations and hospitals require an increasing amount of documentation on medical interventions, patient related data is rarely stored in a structured, processable repository [3]. To overcome this obstacle SDS strongly builds on other data-intensive disciplines, such as data-mining, information theory and statistics, and in general,

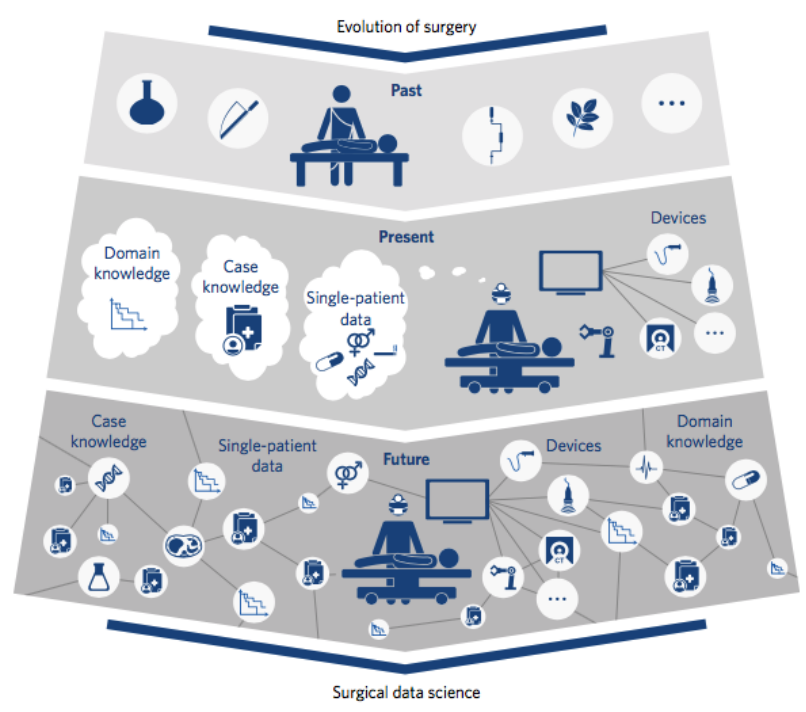

Fig. 1. The development of surgery started with the physician relying on insitu knowledge, and simple tools. Computer Integrated Surgery means, today the available information and instrumentation vastly improved, however the outcome is still dependent on the surgeon's domain knowledge and experience. As next step forward, surgery will become automated in many areas. Patient specific, objective, optimized care will be delivered using Surgical Data Science techniques [2].

follows suit to the trends of industry eventually creating a collaborative, context sensitive environment based on continuous real-time data enabling Surgery 4.0 [4]. With the gathered information SDS enables technologies like smart ORs (employing context aware systems), surgical robotics, decision support systems, speech recognition for OR manipulation [5]. Eventually, improving the human computer interface during surgery will streamline the surgical procedure reducing the cost and improving the outcome of surgeries.

In this article, we give an overview of SDS and its most promising applications. This review is based on online databases (Google Scholar, Medline, Scopus) searched with the keyword: "Surgical Data Science". The search yielded 33 results, from which 2 hits were discarded due to duplications and 6 hits due to irrelevance to the field of SDS. 


\section{Surgical Process Modeling}

Surgical Process (SP) is a term, representing the interventional course realising a surgical goal, for example a specific laparoscopic cholecystectomy procedure to remove the gallbladder. The model of this process is called a Surgical Process Model (SPM), where the model is often represented in a formal or semi-formal manner, focusing on predefined interests [6]. The tool of workflow analysis was originally developed for business applications, and only later applied to the surgical field, where it have been shown to increase safety and efficiency [7].

SPMs can originate either from general concepts being applied to the individual pathology (top down approach), or by analyzing measurement data from surgeries, leading to a generalized concept (bottom up approach).

\section{A. Top-down approach}

The top down approach, what is considered as the traditional way of surgical procedure planning, is based on clinical guidelines and textbooks describing only the very high level concepts of the procedure. With this method, the surgeon's background knowledge will lead to the patient-specific execution of the surgical plan [8]. In the work presented by Munchenberg et al., a system was developed for the preoperative planning and execution of neurosurgical procedures. They employed a haptic interface to interact directly with the patient data, and robotically execute the planned trajectories, using a neurosurgical manipulator [9]. One of the main advantages to the top-down approach is that it is capable of identifying different granularity levels, and accurately describing the surgery with the required detail [10]. While this method is a natural way of representing general knowledge, and is easily understandable for human readers, as the procedure is usually captured by human observers, this representation rarely provide high-level detail about the individual surgeries.

\section{B. Bottom-up approach}

The motivation for examining a bottom-up approach comes from the realization that the above mentioned processes, while capable of representing a reliable surgical plan, the generalized levels are not feasible to be used for quantification. As Neumuth et al. [11] showed, it is possible to create accurate live recordings of surgeries on a detailed, high granularity level. These results showed that the acquired SPM is accurate and reproducible, where the recorded individual SPMs (iSPM) could be used as a basis for process mining techniques. Such techniques were already available, mainly developed for event logs in support of business process model development [12], [13]. Because of several parallel tracks running simultaniously during surgeries, these techniques could not be directly applied to SPMs, and therefore a more generic approach was developed, where the generalized SPM (gSPM) is created using statistical means of the acquired iSPMs [14]. This proved to provide reliable results even when using only 50 iSPMs. In the future, gSPMs could be used as a metric to compare to recorded iSPMs and investigate where and why the
iSPM deviated from the gSPM, gaining valuable knowledge on adverse events, surgical skill. It can be used to estimate operational costs and to compare benefits of different surgical approaches.

\section{ONTOLOGY}

While SPMs do organize the acquired data, they do not necessarily build from a machine readable universal dictionary. For many areas of medicine, international healthcareterminology standards for biomedical data science already exists. These standards are organized into ontologies, such as the Foundational Model of Anatomy [15], Gene Ontology [16] and SNOMED-CT [17]. While these dictionaries do describe the medical background, they do not provide a dictionary for describing interventional medicine. To achieve this goal the OntoSPM international group was formed. The ontology developed by this group (also called OntoSPM) is based on the Basic Formal Ontology (BFO) upper ontology [18], and provides a connection to more specified ontologies, such as LapOntoSPM [19], [20].

\section{SDS IN THE OPERATING THEATER}

Modern Computer Integrated Surgery (CIS) systems provide detailed measurements on the surgical process, however, the data available from the operating computers-for most casesis not captured. By providing a framework for recording, storing and annotating such information (e.g. in the form of SPMs built on Ontologies) SDS provides the ground for a wide variety of clinical applications [21].

\section{A. Context-aware automation and assistance}

The development of CIS started with the development of digital medical imaging, which provided the basis for preoperative planning. These plans could then be used as a model for task execution on robotic platforms, where the robot uses a variety of sensors to update the model to accurately represent the affected anatomical structures. Results from the surgery could be recorded and could directly feed back into a central database to further improve preoperative planning [22]. This concept provides the basis for CIS and is summarized on Fig. 2. One of the major challenges in CIS is the integration of automated procedures into the surgical workflow. Automated workflow monitoring, would allow the development of automated surgical assist systems, and could streamline the surgical process. Such a system was integrated into to the lowlevel control architecture of the ROBODOC [23], but todaymostly because of legal issues-newly developed robotic platforms rarely incorporate such level of autonomy. The use of SPMs could provide a platform, where subtask automationunder the supervision of the surgeon-could be integrated into the surgical procedure, freeing up human assistance during the surgery, eventually lowering healthcare costs. Such application needs to observe the surgical scene, and detect the surgical phases autonomously. To facilitate the development of such applications, both robotic challenges and testing datasets have been presented [24], [25]. On these datasets several 


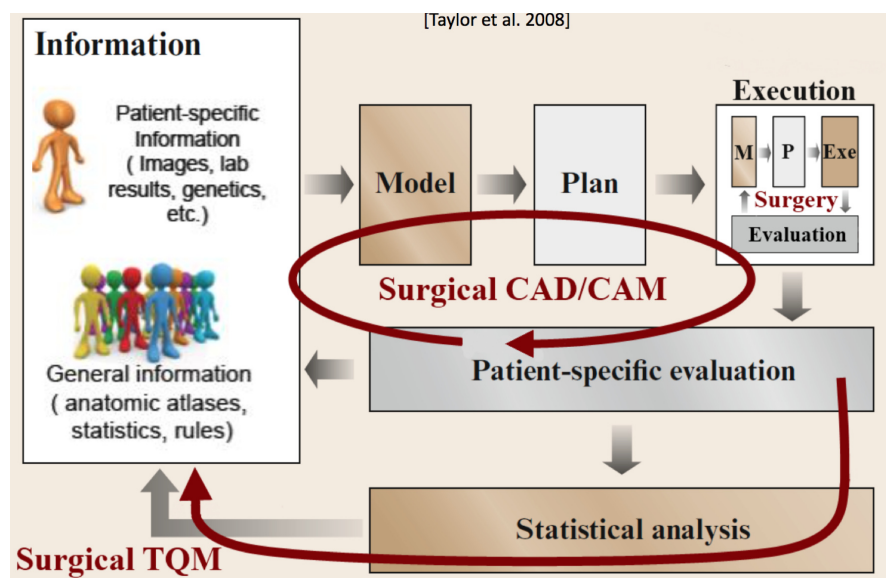

Fig. 2. The concept of Computer Integrated Surgery (CIS) [38] With digital, patient specific data ( e.g. X-ray imaging, Ultrasound, MR, CT) available, surgeons can create detailed pre-operative surgical plans. As these devices are becoming more portable, it is possible to integrate them into the OR. Intraoperative imaging then allows for on the fly re-planning of the procedure. After the procedure, the collected data can be used for statistical analysis, providing information on surgical performance. CAD: Computer-Aided Design CAM: Computer-Aided Manufacturing TQM: Total Quality Management

applications have been developed: for organ [26] and tissue classification [27], [28], as well as for tool recognition [29]. Recently several surgical phase detection algorithms have been presented using Random Forests (RF), Hidden Markov Models (HMM) and deep neural networks [30], [31], [32]. Using the da Vinci Research kit [33], [34], it has been shown in a clinical environment that subtask automation is feasible [35], and SPMs were used to integrate automated robotic intraoperative imaging [36]. Another aspect of workflow monitoring is OR workflow scheduling [37]. Since OR time is one of the most expensive resource of the hospital, by monitoring the workflow, it becomes possible to predict the duration of the surgery, as well as optimizing future procedure plans [14].

\section{B. Decision Support Systems}

One of the main reasons behind the surgical practice being authority based is that more experienced surgeons have a broader understanding of the importance of individual variations in pathology. While experience probably won't be substituted soon, machine learning can process a large amount of pre-recorded data, providing the operating surgeon with decision support, bridging the experience gap, eventually improving patient outcomes. Such systems could also raise attention on areas of patient care, which are generally outside of the surgeon's focus. SDSs analyzing these "unconventional" correlations, could point out important surgical consequences regarding the full record of the patient-care pathway [39].

\section{Surgical Training}

Decision support can not only improve in operation results, but trough simulation, it can play an important role in the education as well [40]. For example, theorem-based semantic reasoning have been used to create a simulation system which is flexible, automated and therefore enables patient-specific scenarios for surgery assistance [41], [42]. Another simulation framework used semantic data to implement a cognitive system which autonomously interacts with ontological knowledge bases and creates individual surgical scenarios [43].

During these simulations, monitoring the workflow of training sessions provides important data on surgical skill and on the individual surgeon's technique [44]. On the low-level (gestures) it has been shown that fine-motor skills correlate with indecisiveness of the surgeon [45], while task level assessment can be used for both skill evaluation and for designing individual training curriculas [46], [47], [48].

\section{Discussion}

Surgical Data Science uses input data from the whole duration of the surgical process and medical care, including the initial symptoms and the long-term outcomes. This approach to surgical interventions require the acquisition and analysis of heterogeneous, multimodal data, which can only be managed if a common framework is developed. This framework can later be used in a multitude of applications including radiation protection planning for interventions [49], analysis software to estimate real-time hypoxemia risk [50], but eventually leads to systems capable of semantically annotating surgical data, performing semantic reasoning and eventually creating context-aware surgical assistance [51]. Ontological knowledge representation shows promising results in this area, however a global framework accepted by professionals and the industry is not available yet. This scale of data collection also raises privacy and confidentiality issues, which requires careful consideration.

SDS is a rapidly developing interdisciplinary field of medicine. While it might seam that development done outside medicine-computer-science and engineering-might solved some of the difficulties in data science, it is challenging to apply those solutions into the surgical field. As this area requires in-depth knowledge of both computer-science and medicine, it seams unavoidable that a new specialty should develop in this field for both academic research and hospital data collection and analysis [52].

\section{ACKNOWLEDGMENT}

The research was supported by the Hungarian OTKA PD 116121 grant.

T.Haidegger is a MTA Boyai Fellow. He is supported through the New National Excellence Program of the Ministry of Human Capacities.

This work has been partially supported by ACMIT (Austrian Center for Medical Innovation and Technology), which is funded within the scope of the COMET (Competence Centers for Excellent Technologies) program of the Austrian Government.

We acknowledge the financial support of this work by the Hungarian State and the European Union under the EFOP3.6.1-16-2016-00010 project. 


\section{REFERENCES}

[1] D. L. Sackett, "Evidence-based medicine," Seminars in Perinatology, vol. 21, no. 1, pp. 3-5, Feb. 1997

[2] L. Maier-Hein, S. S. Vedula, S. Speidel, N. Navab, R. Kikinis, A. Park, M. Eisenmann, H. Feussner, G. Forestier, S. Giannarou, M. Hashizume, D. Katic, H. Kenngott, M. Kranzfelder, A. Malpani, K. März, T. Neumuth, N. Padoy, C. Pugh, N. Schoch, D. Stoyanov, R. Taylor, M. Wagner, G. D. Hager, and P. Jannin, "Surgical data science for next-generation interventions," Nature Biomedical Engineering, vol. 1, no. 9, pp. 691696, Sep. 2017.

[3] D. Krefting, M. Vossberg, A. Hoheisel, and T. Tolxdorff, "Simplified implementation of medical image processing algorithms into a grid using a workflow management system," Future Generation Computer Systems, vol. 26, no. 4, pp. 681-684, Apr. 2010.

[4] H. Feußner and A. Park, "Surgery 4.0: The natural culmination of the industrial revolution?" Innovative Surgical Sciences, vol. 2, no. 3, pp. 105-108, 2017.

[5] J. Miehle, D. Ostler, N. Gerstenlauer, and W. Minker, "The next step: Intelligent digital assistance for clinical operating rooms," Innovative Surgical Sciences, vol. 2, no. 3, pp. 159-161, 2017.

[6] T. Neumuth, S. Schumann, G. Strauß, and P. Jannin, "Visualization Options for Surgical Workflows," International Journal of Computer Assisted Radiology and Surgery, pp. 438-440, 2006.

[7] C. F. Dickhaus, C. Burghart, C. Tempany, A. D’Amico, S. Haker, R. Kikinis, and H. Woern, "Workflow modeling and analysis of computer guided prostate brachytherapy under MR imaging control." Studies in health technology and informatics, vol. 98, pp. 72-74, 2004.

[8] C. M. Townsend, R. D. Beauchamp, B. M. Evers, and K. L. Mattox, Sabiston Textbook of Surgery E-Book. Elsevier Health Sciences, Apr. 2016.

[9] J. Munchenberg, J. Brief, J. Raczkowsky, H. Worn, S. Hassfeld, and J. Muhling, "Operation planning of robot supported surgical interventions," in Proceedings. 2000 IEEE/RSJ International Conference on Intelligent Robots and Systems (IROS 2000), vol. 1, 2000, pp. 547-552 vol.1.

[10] C. MacKenzie, Ibbotson AJ, Cao CGL, and Lomax A, "Hierarchical decomposition of laparoscopic surgery: A human factors approach to investigating the operating room environment." Minim Invasive Ther Allied Technol, vol. 10, no. 3, pp. 121-128, 2001.

[11] T. Neumuth, P. Jannin, G. Strauss, J. Meixensberger, and O. Burgert, "Validation of knowledge acquisition for surgical process models," Journal of the American Medical Informatics Association: JAMIA, vol. 16, no. 1, pp. 72-80, 2009 Jan-Feb.

[12] W. M. P. van der Aalst, B. F. van Dongen, J. Herbst, L. Maruster, G. Schimm, and A. J. M. M. Weijters, "Workflow mining: A survey of issues and approaches," Data \& Knowledge Engineering, vol. 47, no. 2, pp. 237-267, Nov. 2003.

[13] G. Schimm, "Mining exact models of concurrent workflows," Computers in Industry, vol. 53, no. 3, pp. 265-281, Apr. 2004.

[14] T. Neumuth, P. Jannin, J. Schlomberg, J. Meixensberger, P. Wiedemann, and O. Burgert, "Analysis of surgical intervention populations using generic surgical process models," International Journal of Computer Assisted Radiology and Surgery, vol. 6, no. 1, pp. 59-71, Jan. 2011.

[15] C. Rosse and J. L. V. Mejino, "A reference ontology for biomedical informatics: The Foundational Model of Anatomy," Journal of Biomedical Informatics, vol. 36, no. 6, pp. 478-500, Dec. 2003.

[16] M. Ashburner, C. A. Ball, J. A. Blake, D. Botstein, H. Butler, J. M. Cherry, A. P. Davis, K. Dolinski, S. S. Dwight, J. T. Eppig, M. A. Harris, D. P. Hill, L. Issel-Tarver, A. Kasarskis, S. Lewis, J. C. Matese, J. E. Richardson, M. Ringwald, G. M. Rubin, and G. Sherlock, "Gene ontology: Tool for the unification of biology. The Gene Ontology Consortium," Nature Genetics, vol. 25, no. 1, pp. 25-29, May 2000.

[17] D. Lee, N. de Keizer, F. Lau, and R. Cornet, "Literature review of SNOMED CT use," Journal of the American Medical Informatics Association, vol. 21, no. e1, pp. e11-e19, Feb. 2014.

[18] R. Arp and B. Smith, "Function, Role and Disposition in Basic Formal Ontology," in Nature Precedings, 2008.

[19] D. Katic, Chantel Julliard, Anna-Laura Wekerle, Hannes Kenngott, Beat Peter Müller-Stich, Rüdiger Dillmann, Stephanie Speidel, Pierre Jannin, and Bernard Gibaud, "LapOntoSPM: An ontology for laparoscopic surgeries and its application to surgical phase recognition," Int J Comput Assist Radiol Surg, vol. 10, no. 9, pp. 1427-1434, Jun. 2015.
[20] D. Katić, M. Maleshkova, S. Engelhardt, I. Wolf, K. März, L. MaierHein, M. Nolden, M. Wagner, H. Kenngott, B. P. Müller-Stich, R. Dillmann, and S. Speidel, "What does it all mean? Capturing Semantics of Surgical Data and Algorithms with Ontologies," arXiv:1705.07747 [cs], p. 4, May 2017.

[21] S. S. Vedula, M. Ishii, and G. D. Hager, "Perspectives on Surgical Data Science," arXiv:1610.04276 [cs], p. 4, Oct. 2016.

[22] Á. Takács, D. Á. Nagy, I. Rudas, and T. Haidegger, "Origins of Surgical Robotics: From Space to the Operating Room," ACTA POLYTECHNICA HUNGARICA, vol. 13, no. 1, pp. 13-30, 2016.

[23] P. Kazanzides, J. Zuhars, B. Mittelstadt, B. Williamson, P. Cain, F. Smith, L. Rose, and B. Musits, "Architecture of a surgical robot," in 1992 IEEE International Conference on Systems, Man, and Cybernetics, Oct. 1992, pp. 1624-1629 vol.2.

[24] Y. Gao, S. S. Vedula, C. E. Reiley, N. Ahmidi, B. Varadarajan, H. C. Lin, L. Tao, L. Zappella, B. Béjar, and D. D. Yuh, "JHU-ISI gesture and skill assessment working set (JIGSAWS): A surgical activity dataset for human motion modeling," in MICCAI Workshop: M2CAI, vol. 3, 2014.

[25] R. Stauder, D. Ostler, M. Kranzfelder, S. Koller, H. Feußner, and N. Navab, "The TUM LapChole dataset for the M2CAI 2016 workflow challenge," arXiv:1610.09278 [cs], p. 6, Oct. 2016.

[26] S. Moccia, S. J. Wirkert, H. Kenngott, A. S. Vemuri, M. Apitz, B. Mayer, E. De Momi, L. S. Mattos, and L. Maier-Hein, "UncertaintyAware Organ Classification for Surgical Data Science Applications in Laparoscopy," arXiv:1706.07002 [cs], p. 7, Jun. 2017.

[27] S. Moccia, E. D. Momi, M. Guarnaschelli, M. Savazzi, A. Laborai, L. Guastini, G. Peretti, and L. S. Mattos, "Confident texture-based laryngeal tissue classification for early stage diagnosis support," Journal of Medical Imaging, vol. 4, no. 3, p. 034502, Sep. 2017.

[28] M. L. M. Savazzi, M. Guarnaschelli, and D. M. Elena, "Machine learning for tissue classification in laryngeal endoscopic videos," Thesis: Laurea Magistrale / Specialistica, Politechnico Milano, Milano, Apr. 2017.

[29] L. C. Garcia-Peraza-Herrera, W. Li, L. Fidon, C. Gruijthuijsen, A. Devreker, G. Attilakos, J. Deprest, E. V. Poorten, D. Stoyanov, T. Vercauteren, and S. Ourselin, "ToolNet: Holistically-Nested Real-Time Segmentation of Robotic Surgical Tools," arXiv:1706.08126 [cs], p. 6, Jun. 2017.

[30] R. Stauder, D. Ostler, T. Vogel, D. Wilhelm, S. Koller, M. Kranzfelder, and N. Navab, "Surgical data processing for smart intraoperative assistance systems," Innovative Surgical Sciences, vol. 2, no. 3, pp. 145-152, 2017.

[31] R. Stauder, E. Kayis, and N. Navab, "Learning-based Surgical Workflow Detection from Intra-Operative Signals," arXiv:1706.00587 [cs], Jun. 2017.

[32] N. Ahmidi, L. Tao, S. Sefati, Y. Gao, C. Lea, B. B. Haro, L. Zappella, S. Khudanpur, R. Vidal, and G. D. Hager, "A Dataset and Benchmarks for Segmentation and Recognition of Gestures in Robotic Surgery," IEEE Transactions on Biomedical Engineering, vol. 64, no. 9, pp. 20252041, Sep. 2017.

[33] P. Kazanzides, Zihan Chen, Anton Deguet, Gregory S. Fischer, Russell H. Taylor, and Simon P. DiMaio, "An open-source research kit for the da Vinciß Surgical System," in 2014 IEEE International Conference on Robotics and Automation (ICRA). Hong Kong, China: Institute of Electrical and Electronics Engineers Inc., Sep. 2014, pp. 6434-6439.

[34] Á. Takács, I. Rudas, and T. Haidegger, "Open-Source Research Platforms and System Integration in Modern Surgical Robotics," Acta Universitatis Sapientiae Electrical and Mechanical Engineering, vol. 14, no. 6, pp. 20-34, 2015.

[35] A. Murali, S. Sen, B. Kehoe, A. Garg, S. McFarland, S. Patil, W. D. Boyd, S. Lim, P. Abbeel, and K. Goldberg, "Learning by observation for surgical subtasks: Multilateral cutting of 3D viscoelastic and 2D Orthotropic Tissue Phantoms," in 2015 IEEE International Conference on Robotics and Automation (ICRA), May 2015, pp. 1202-1209.

[36] N. Navab, M. Fellow, C. Hennersperger, B. Frisch, and B. Fürst, "Personalized, relevance-based Multimodal Robotic Imaging and augmented reality for Computer Assisted Interventions," Medical Image Analysis, vol. 33, no. Supplement C, pp. 64-71, Oct. 2016.

[37] S. Franke, J. Meixensberger, and T. Neumuth, "Intervention time prediction from surgical low-level tasks," Journal of Biomedical Informatics, vol. 46, no. 1, pp. 152-159, Feb. 2013.

[38] Bruno Siciliano, "Medical robots and systems ch.52," in Springer Handbook of Robotics. Berlin: Springer, 2008. 
[39] M. Wagner, T. Weller, L.-M. Ternes, R. Rempel, M. Maleshkova, Y. Sure-Vetter, and H. Kenngott, "Bringing Data-Driven Process Analysis into Surgical Practice - the Surgical Process Analyzer," in Proceedings Surgical Data Science. Heidelberg: Springer, Jun. 2016, p. 6.

[40] N. Schoch, F. Kißler, M. Stoll, S. Engelhardt, R. de Simone, I. Wolf, R. Bendl, and V. Heuveline, "Comprehensive patient-specific information preprocessing for cardiac surgery simulations," International Journal of Computer Assisted Radiology and Surgery, vol. 11, no. 6, pp. 1051-1059, Jun. 2016.

[41] N. Schoch, S. Speidel, Y. Sure-Vetter, and V. Heuveline, "Towards semantic simulation for patient-specific surgery assistance," OnlineProceedings of Surgical Data Science, p. 6, 2016.

[42] N. Schoch and V. Heuveline, "Towards an Intelligent Framework for Personalized Simulation-enhanced Surgery Assistance: Linking a Simulation Ontology to a Reinforcement Learning Algorithm for Calibration of Numerical Simulations," Preprint Series of the Engineering Mathematics and Computing Lab, no. 5, Oct. 2017.

[43] Nicolai Schoch and Vincent Heuveline, "Towards Cognition-Guided Patient-Specific FEM-Based Cardiac Surgery Simulation," in Functional Imaging and Modelling of the Heart, ser. Lecture Notes in Computer Science. Springer, Cham, Jun. 2017, pp. 115-126.

[44] G. Forestier, F. Petitjean, P. Senin, L. Riffaud, P.-L. Henaux, and P. Jannin, "Finding discriminative and interpretable patterns in sequences of surgical activities," Artificial Intelligence in Medicine, vol. 82 Supplement C, pp. 11-19, Oct. 2017.

[45] H. Mohamadipanah, C. Parthiban, L. Maulson, S. Laufer, D. Rutherford, K. Forsyth, S. DiMarco, and C. Pugh, "Is Indecisiveness Linked to Hand Movements in Performing Laparoscopic Ventral Hernia Repair?" Heidelberg, Germany, Jun. 2016, p. 6.

[46] C. E. Reiley and G. D. Hager, "Task versus Subtask Surgical Skill Evaluation of Robotic Minimally Invasive Surgery," in LNCS 5761, vol. Part I. Springer-Verlag Berlin Heidelberg, 2009, pp. 435-442.

[47] C. C. Greenberg, H. N. Ghousseini, S. R. Quamme Pavuluri, H. L. Beasley, and D. A. Wiegmann, "Surgical coaching for individual performance improvement," Annals of Surgery, vol. 261, no. 1, pp. 32-34, Jan. 2015.

[48] S. S. Vedula, M. Ishii, and G. D. Hager, "Objective Assessment of Surgical Technical Skill and Competency in the Operating Room," Annual Review of Biomedical Engineering, vol. 19, no. 1, pp. 301-325, 2017.

[49] N. Loy Rodas, M. De Mathelin, and N. Padoy, "Radiation safety in tomorrow's hospital," Heidelberg, Germany, Jun. 2016.

[50] S. M. Lundberg, B. Nair, M. S. Vavilala, M. Horibe, M. J. Eisses, T. Adams, D. E. Liston, D. K.-W. Low, S.-F. Newman, J. Kim, and S.-I. Lee, "Explainable machine learning predictions to help anesthesiologists prevent hypoxemia during surgery," bioRxiv, p. 206540, Oct. 2017.

[51] A. Fetzer, J. Metzger, D. Katic, K. März, M. Wagner, P. Philipp, S. Engelhardt, T. Weller, S. Zelzer, A. M. Franz, N. Schoch, V. Heuveline, M. Maleshkova, A. Rettinger, S. Speidel, I. Wolf, H. Kenngott, A. Mehrabi, B. P. Müller-Stich, L. Maier-Hein, H.-P. Meinzer, and M. Nolden, "Towards an open-source semantic data infrastructure for integrating clinical and scientific data in cognition-guided surgery," vol. 9789. International Society for Optics and Photonics, Mar. 2016, p. 978900 .

[52] L. Maier-Hein, S. Vedula, S. Speidel, N. Navab, R. Kikinis, A. Park, M. Eisenmann, H. Feussner, G. Forestier, S. Giannarou, M. Hashizume, D. Katic, H. Kenngott, M. Kranzfelder, A. Malpani, K. März, T. Neumuth, N. Padoy, C. Pugh, N. Schoch, D. Stoyanov, R. Taylor, M. Wagner, G. D. Hager, and P. Jannin, "Surgical Data Science: Enabling NextGeneration Surgery," arXiv:1701.06482 [cs], p. 10, Jan. 2017. 
D. Ákos Nagy et al. • Surgical Data Science, An Emerging Field of Medicine 